\title{
Exploring Knowledge Based Creativity: A Systems Approach Perspective
}

\author{
Joel Njase, Edward Lambert \\ School of Business \& Economics, Atlantic International University, Honolulu, USA \\ Email: njasejoel@gmail.com
}

How to cite this paper: Njase, J., \& Lambert, E. (2020). Exploring Knowledge Based Creativity: A Systems Approach Perspective. Open Journal of Business and Management, 8 , 1831-1853. https://doi.org/10.4236/ojbm.2020.84112

Received: May 26, 2020

Accepted: July 27, 2020

Published: July 30, 2020

Copyright (c) 2020 by author(s) and Scientific Research Publishing Inc. This work is licensed under the Creative Commons Attribution International License (CC BY 4.0).

http://creativecommons.org/licenses/by/4.0/

(c) (i) Open Access

\begin{abstract}
Creativity has played a major role in innovation advancement in various industries across the globe. In this present research, enhancement of Creativity Development Process (CDP) in business, through an eye of Systems Approach Perspective (SAP) is under-pinned. Notwithstanding, actuating the deliberate development of skills, roles and competencies needed for effective performance of work, promotes shaping business values and culture. Therefore, the aim of this study through action research is to enhance analytical creative development process, necessary for attaining high quality knowledge base creativity for business innovation. Previous research in development of creativity based on knowledge, focused on strengthening process of information collection and evidence handling to solve a problem. Further, research dealt with role of creativity in innovation process, and other focused of categories of creativity such as primary creativity or inspiration and secondary creativity or elaboration. Using a 3-point Matrix scale, research showed that decision process using systems analysis approach enhanced creativity development process. In this regard, the immense contribution of applying System Analysis Approach (SAAP) to optimize capacity in decision making on choice of idea, through sustainable creative thinking and innovation cannot be under-estimated.
\end{abstract}

\section{Keywords}

Knowledge-Based Creativity, Systems Analysis Approach, Development Process, Decision Making

\section{Introduction}

The determination of best course of action in both social and economic development has witnessed diverse decision-making methods. Despite perceived bias resulting in false representation of support for given decision methods such as 
Naturalistic decision Model, multi-attribute Utility analysis, Decision Analysis (Oroszi, 2020), improving decision making performance in current competitive world is vital. Therefore, the application of decision process such as System Analysis Approach to Creativity Development Process (CDP) to generate high quality perspectives needs exploring in research. In this regard, the need to enhance creativity and possible innovation drive necessary to do business cannot be over-emphasized.

The recent past has witnessed creative thinking becoming part of core business skills, application of creative solutions to the problems, and taking advantage of available opportunities, hence providing innovative impetus necessary for any national survival and success (Scarborough \& Cornwell, 2016). Notwithstanding, actuating the deliberate development of skills, roles and competencies needed for effective performance of work, promotes shaping business values and culture. Therefore, enhancing analytical development tools is likely to result in high quality knowledge base creativity necessary for business innovation. Previous research in development of creativity based on knowledge, focused on strengthening process of information collection and evidence handling to solve a problem. In this regard, Scarborough \& Cornwell (2016) established seven steps necessary to develop creativity which included: preparation, investigation, transformation, incubation, illumination, verification and implementation phases. Further, research dealt with role of creativity in innovation process, and other focused categories of creativity such as primary creativity or inspiration and secondary creativity or elaboration. Leadership expert Warren Bennis says, "Today's successful companies live and die according to the quality of their ideas." In order to enhance quality of ideas numerous techniques such as Brainstorming, Mind Mapping, Force-Field Analysis, TRIZ and Rapid Prototyping have been pursued in previous research (Scarborough \& Cornwell, 2016).

In this present research, enhancement of Creativity Development Process (CDP) in business, through an eye of Systems Approach Perspective (SAP) is under-pinned. A process is defined as "a sequence of interrelated activities that proceed in time" (Dandy et al., 2008). Action Research is proposed through unstructured interviews (Fontana \& Frey, 2005) with selected entrepreneurs, document reviews and observations (Kawalich, 2005), and focus groups (Barbour, 2005). In this regard, Labi (2014) retorted the need for solid set of skills in other disciplines, to accelerate the infusion of "system" perspective in traditional engineering fields. For this reason, business success in the current COVID-19 pandemic, global social and economic challenges is no exception to this rule. Therefore, the immense contribution of applying System Analysis Approach (SAAP) to optimize capacity in decision making on choice of idea, through sustainable creative thinking and innovation cannot be under-estimated.

\subsection{Statement of the Problem}

Innovation must be a constant process because most ideas don't work and most 
innovations fail (Scarborough \& Cornwell, 2016). However, there can be no innovation without creativity based on knowledge.

\subsection{Main Objective}

To apply systems approach analysis in creative thinking development process, in order to optimize entrepreneurial capacity in decision making on choice of a new idea necessary for innovative drive.

\subsection{Specific Objectives}

1) Improve decision making in creative thinking development process through SAAP,

2) Identify factors necessary for enhancement of knowledge-based creativity, and

3) Propose recommendations to problem analysis in creativity development process.

\subsection{Research Questions}

1) How can Systems Analysis Approach be applied to enhance creative development process?

2) What factors affect choice of new idea in the creativity development process?

3) What is the best way to conduct problem analysis in creativity development process.

\subsection{Research Hypothesis}

In this paper, the author will apply Systems analysis approach to enhance creativity development process, in decision making on choice of an idea or solution to a problem.

$\mathrm{H}$ Choice of a new idea or creativity based on knowledge is influenced by decision process.

\subsection{Research Justification}

Increasing societal demand for high quality services or products across the globe coupled with diminishing natural resources, has necessitated constant innovation drive in diverse industries. Notwithstanding, this calls for a parallel enhanced creativity development process based on knowledge.

\subsection{Methodology}

The selection of research approach and data collection methods depends on the facilities available, period, researcher skills and other costs and associated resources needed (Frenchtling \& Sharp, 1997; Sekaran, 2003). In this present research, enhancement of Creativity Development Process (CDP) in business, through an eye of Systems Approach Perspective (SAP) is under-pinned. A pro- 
cess is defined as "a sequence of interrelated activities that proceed in time" (Dandy et al., 2008). Action Research was under-taken through unstructured interviews (Fontana \& Frey, 2005) with selected entrepreneurs in Muchinga Province-Zambia, document reviews and observations (Kawalich, 2005), and two separate focus groups (Barbour, 2005).

\subsubsection{Participants}

A total number of forty (40) entrepreneurs mostly in service and retail business category participated, representing $42 \%$ of entrepreneurs in Nakonde district under the new and most recently created tenth (10) Province in the great Republic of Zambia. The female represented $47.5 \%$, while male represented $52.5 \%$ of the total participants. Some of these participants were drawn from various government ministries and banks, but involved in small and medium scale businesses. Those in formal employment aforementioned represented 62.5\%. Further, a total of fifteen (15) participants representing 37.5\% run very successful business, seven (7) were average performers in business, and eighteen (18) were straggling representing $45 \%$. Table 2 , demonstrates the demographic representation based on age, gender and educational level. On the other hand, two (2) main groups were formed and each group was subdivided into five (5) sub-groups each with four (4) members. Each sub-group was tasked to develop new perspectives (ideas), to create social and economic development by transforming their own businesses. Nakonde is situated in Muchinga province endowed with high value natural resources and great business opportunities being a border town with Tanzanian.

\subsubsection{Focus Groups}

In enhancing creativity development process involving the two independent focus groups, six (6) categories based on the research model were considered, in exploiting the decision process in creativity development factors on measure on: need identification, business environment understanding, opportunity identification skills, value analysis, expectation in respect to benefits to society, and uniqueness of the perspective was undertaken. This process was critical in development of perspective's value, overall goals, goals, objectives measure of effectives and subsequent scoring of two focus groups (Table 1 ).

Group 1, was exposed to Systems Analysis Approach considering level of

Table 1. Focus groups.

\begin{tabular}{|c|c|c|c|c|c|}
\hline \multicolumn{6}{|c|}{ Entrepreneurial focus groups-Muchinga Province } \\
\hline \multirow{2}{*}{$\begin{array}{c}\text { No. } \\
1\end{array}$} & \multirow{2}{*}{$\begin{array}{c}\text { category } \\
\text { Age }\end{array}$} & \multicolumn{2}{|c|}{ group 1} & \multicolumn{2}{|c|}{ group 2} \\
\hline & & \multicolumn{2}{|c|}{$18-25$} & \multicolumn{2}{|c|}{$26-35$} \\
\hline \multirow[b]{2}{*}{2} & \multirow[b]{2}{*}{ Gender } & Male & Female & Male & Female \\
\hline & & 8 & 12 & 13 & 7 \\
\hline 3 & Education level & High Sc & Vocation & & sity \\
\hline
\end{tabular}


education and was used as a control group during the research period. This group was not allowed to use any method in the creativity development process apart from aforesaid method.

Group 2, with high level of education was not exposed to the process but given the same problem as the first group. The shared understanding for this group was to allow free discussions in this group and ensure exchange of information. However, the composition of this group with great managerial skills and competencies was to generate perspectives with any method.

Figure 1 \& Figure 2 show participants' gender distribution and percentage business performance of the participants in their private life respectively.

\subsubsection{Approach}

Unstructured questions based on exploiting our research model for enhancement of creativity development process namely: Problem Analysis, Knowledge, Creative "SPARK", Decision Process, Validation, and New Idea. The questions were presented to various sub-groups independently and then to each main group separately. However, the concept of shared understanding on the subject centered on group assessment on their ability to deal work around the given task

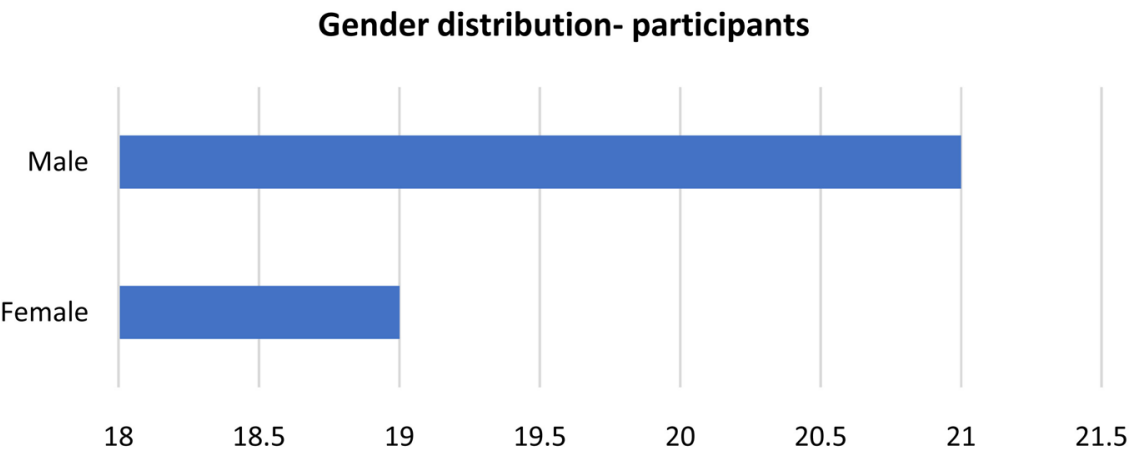

Figure 1. Gender distribution (participants).

Participants business performance

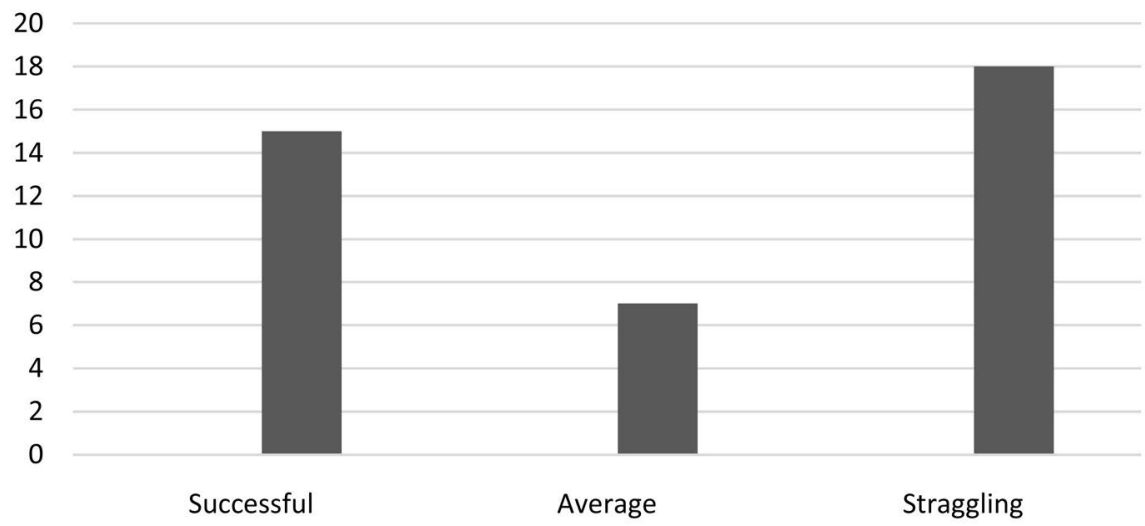

- Series1 - Series2

Figure 2. Individual business performance (participants). 
and measure of capabilities to do need identification, interrogation of business environment, business opportunity identification, value analysis base on value awareness, expectations in terms of benefits, and uniqueness of the developed perspectives. Two level answer based on observation of the groups was a Yes or No in under-taking a comparative task-fit achievement (Table 2).

In enhancing creativity development process involving the two independent focus groups, six (6) categories based on the research model were considered and in exploiting the decision process in creativity development factors on measure on: need identification, business environment understanding, opportunity identification skills, value analysis, expectation in respect to benefits to society, and uniqueness of the perspective was undertaken. This process was critical in development of perspective's value, overall goals, goals, objectives measure of effectives and subsequent scoring of two focus groups.

\subsection{Proposed Creativity Development Process (CDP)}

Decision making, (Rilling \& Sanfey, 2011) is the process of selecting and implementing a program or strategy based on individual values and preferences among multiple alternatives. In our present research decision making is the selection and implementation of alternative perspectives in the creative development process in order to create a new idea. Therefore, the following steps based on systems analysis approach (SAAP) were pursued to enhance creativity development process and included the following:

\section{1) Problem Identification \& Analysis}

The first step in the Creativity Development Process (CDP), using systems analysis approach involved, problem identification and exploiting of knowledge based on skills, capabilities, focused on societal needs, expected value addition, opportunity and expected satisfaction. Notwithstanding, knowledge on factors affecting innovation diffusion was important to provide a balanced systems approach to CDP. This resulted in clearly defined decision gaps necessary to assist

Table 2. Indicate outcome from observation during focus group discussions.

\begin{tabular}{|c|c|c|c|c|c|c|}
\hline \multicolumn{7}{|c|}{ Entrepreneurial Focus Groups (based on research model) } \\
\hline \multirow{3}{*}{ No. } & \multirow{3}{*}{ Category } & \multirow{3}{*}{ Measure } & \multicolumn{4}{|c|}{ Evaluation } \\
\hline & & & \multicolumn{2}{|c|}{ Group 2} & \multicolumn{2}{|c|}{ Group 2} \\
\hline & & & Yes & No & Yes & No \\
\hline 1 & Problem analysis & Need identification & $\mathrm{x}$ & & $\mathrm{x}$ & \\
\hline 2 & Knowledge & Business environment & & $\mathrm{x}$ & $\mathrm{x}$ & \\
\hline 3 & Creative "SPARK" & Opportunity identification & $\mathrm{x}$ & & & $\mathrm{x}$ \\
\hline 4 & Decision Process & Value analysis & $\mathrm{x}$ & & $\mathrm{x}$ & \\
\hline 5 & Validation & Expectation & $\mathrm{x}$ & & & $\mathrm{x}$ \\
\hline 6 & New idea & Uniqueness & $\mathrm{x}$ & & $\mathrm{x}$ & \\
\hline
\end{tabular}


in determination of value centered objectives in creative process. On the other hand, development of conceptual skills which are mental abilities to see how various factors in a given situation fit together and interact is critical in creativity development process. In this regard, problem analysis uses of explicit and measurable criteria to evaluate initial confronted and congregate them into multi-dimensional viewpoints.

\section{2) Objective Analysis}

Based on primary creativity drivers such as societal needs, expected value addition, opportunity and expected satisfaction, objectives are set to realize a coined approach in idea development. With an interrogative mind diverse views are narrowed to specific idea, through an eye to system analysis approach. Further, creativity determinants based on type of innovation cannot be under-estimated on their effect on creativity development process.

\section{3) Establishment of View-Points}

Philosophy is the value system of an individual, group or organization, which can be expressed in ethical context as means of production for goods and services. This is where, culture which is the way of life style (Meredith et al., 2009) associated with a group of people and involves all aspects of living including attitudes, skills, tools and habits commands a significant effect on creativity development process. In this regard, various viewpoints in the creativity development process focused on philosophy and theory relating to multi-dimensional approach to the problem, are established from set objectives based on secondary creativity drivers. The secondary creativity drivers maybe as a result of a) reactive innovation based on response to customers feedback or changing market conditions; b) proactive innovation based on spotting opportunities on which to capitalize; c) revolutionary innovation based on creating market-changing disruptive breakthroughs; d) evolutionary innovation based on market sustainability.

\section{4) Viewpoints Analysis}

According to Eltinary et al. (2013) in quoting Olson \& Slate (2002) retorted that performance measurement is a significant component of management control process of any organization. In light of this; measurement of different viewpoints of the main idea, weighted using a non-numerical 3-point scale is an important control process in creativity development process. Dependent on hierarchy of desired system outcomes (abstract system). Based on four domains of system theory, an interrogation of the various perspectives of the main idea considering each perspective's value, overall goals, goals, objectives measure of effectives.

\section{5) Interpretation}

Interpret each viewpoint in terms of results and consider next viewpoint (idea). During this phase consideration on weighted contribution to develop an innovative drive and subsequent potential innovation diffusion cannot be under-estimated. In technological diffusion process various adoption and acceptance theories (Sai, 2018) have been underpinned and include: a) theory of 
Reasoned Action (TRA) based on person's intentions, b) the Theory of Planned Behavior (TPB) which extends the TRA and perceived behavioral construct, c) Technological Acceptance Model (TAM) based on attitude on perceived usefulness, d) the theory of Consumption values, and e) the theory of Innovation Diffusion Theory (IDT) which holds that the adoption rate depends on its relative advantage, compatibility, triability, observability, and complexity among others. This stage is vital in the creativity development process to get high quality viewpoints.

\section{6) Viewpoint Selection}

Based on the proposed 3-point matrix scale of Table 4, scores on each perspective of the main idea are added and aggregated. Perspective with the highest score is selected.

\section{7) Implementation \& Evaluation}

Notwithstanding, the validation of selected perspective (idea) is reviewed using (Scarborough \& Cornwell, 2016) seven steps necessary to develop creativity which included: preparation, investigation, transformation, incubation, illumination, verification and implementation. Therefore, a new idea is borne and coined as an integrated perspective necessary to create an innovative drive seen in current research as part of creativity development process final stage. An idea without value, goals, objectives, and measure of effectiveness on resolving a problem is not creativity.

\subsection{Findings}

Enhanced decision-making process in creativity development process, can improve quality of creativity. Further, adequate knowledge based on value-centered creativity, promotes spotting of opportunities. Notwithstanding, systems analysis approach cannot be underestimated as an essential tool, for accelerating the creative potential in regard to innovative drive critical for power to solve problems.

\section{Review of Literature}

\subsection{Knowledge-Based Creativity in Business: A Systems Approach Perspective}

The study of development process in creativity based on knowledge, is an important research area with great potential to bring vivid and diverse global services and goods. It demands interest in creative mental patterns and understanding specific fields in the environment, especially steps involved in realizing the aforementioned. Figure 3 illustrates a proposed Knowledge based creativity-model. Creativity expert Teresa Amabile identified three components of creativity which included expertise, creative thinking skills, and motivation (Scarborough \& Cornwell, 2016). Notwithstanding, the great ease of spread in respect to COVID-19 and late global integrated response approach to fight the health pandemic, has exposed knowledge gaps in human creativity development. 


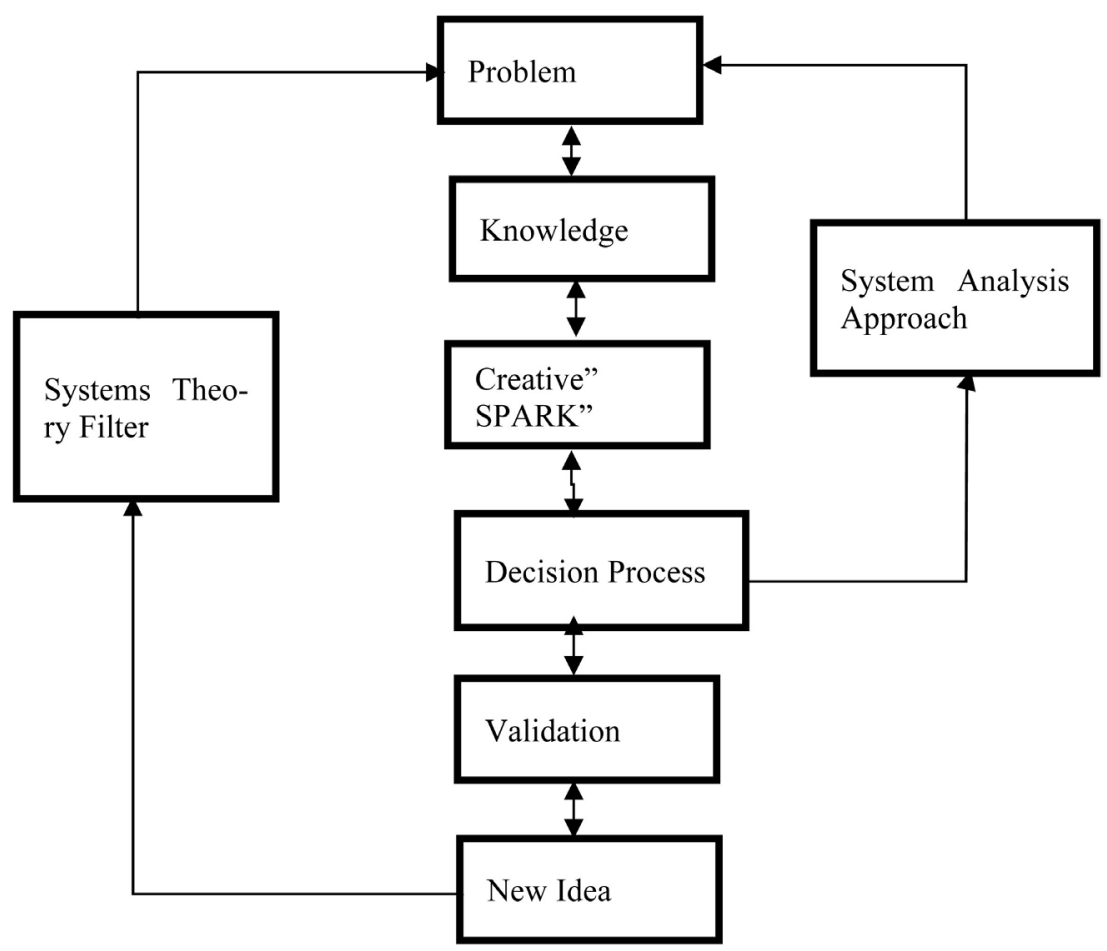

Figure 3. Knowledge-based creativity (KBC) - model.

Hence, failing to predict potential scale of threat, especially on human social and economic activities. This is where, the call to multi-dimensional focus to knowledge-based creativity in business, focused on systems approach perspective is the new global recourse to such challenges. In this regard, preparation of the mind for creative thinking based on knowledge is important and can be through formal education, on-the-job training, work experience, and other learning opportunities. The need to study the problem and understanding its basic components demands careful investigations and cannot be under-estimated. Figure 4, illustrates summary on figure, and shows that with knowledge-controlled process we can achieve creativity.

Since the present research is focused on enhancement of creativity development process (CDP) in business, through an eye of Systems Approach Perspective (SAP), it is important to understand the process dimension in creativity. A process is defined as "a sequence of interrelated activities that proceed in time" (Dandy et al., 2008). In light of the aforesaid, whether creativity is spontaneous or discrete in form, it will still be bound by time. Further, whether creative process occurs concurrently or in multi-process form is yet another area of knowledge-based creativity development process.

Therefore, taking knowledge-based creativity as a system with three components of creativity, which included expertise, creative thinking skills, and motivation (Scarborough \& Cornwell, 2016) as identified by creativity expert Teresa Amabile, presents a foundation of shared understanding on creativity study. A system is a selection of elements, relationships, and procedures to achieve a specific 


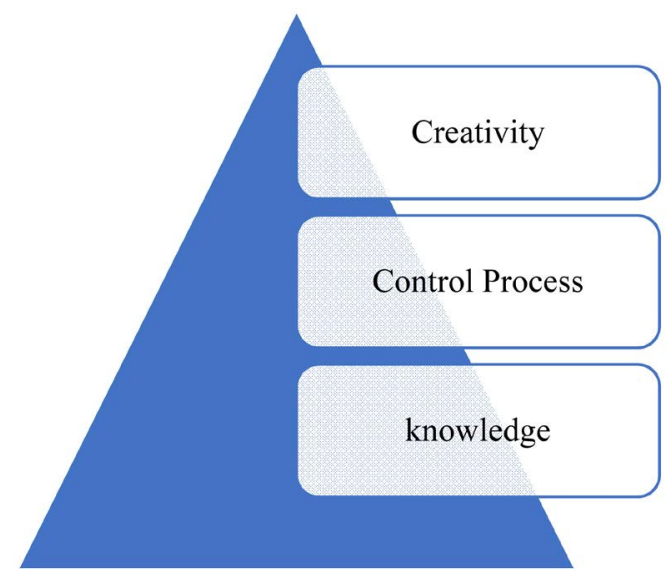

Figure 4. Creativity based on knowledge-model.

purpose (Wortman \& Luthan, 1969), where purpose is reason for existence. Other scholars retorted that, a system is a group of interacting, interrelated, or interdependent elements forming a complex whole (Collins, 2009). Further, deliberate development of skills, roles and competencies needed to effectively perform work, is promoted through shaping business values and culture. This is done through gaining experience in decision making and leading by example (modeling. In this way, business's direct and support work to be performed effectively, and preparation of a business to deal with threats and opportunities in its external environment by entrepreneurs is established. Therefore, based on systems analysis perspective, required knowledge to create a "SPARK" must clearly underscore the scope of the problem in terms of need, value, form, shape, cause, effect, as well as general environmental characteristics and dynamics. Further, the industrial or service sector must be well understood. Not only that but also associated objectives to knowledge-based creativity to enhance quality of creative "SPARK".

\subsection{Creative "SPARK"}

According to Scarborough \& Cornwell (2016) creativity is the power to deal with a problem and taking opportunities, using new ways and ideas. In view of operational definition of creativity (Wu et al., 2014) expounds that it's a multidimensional construct, located on the border between cognition and personality with most researchers agreeing that it cannot be defined conceptually but operationally. Creative "SPARK" involves the analysis or transformative process of collected information, through convergent and divergent thinking to yield a new thought process necessary to creation of a new ideal or solution.

Therefore, Creative "SPARK" is the spontaneous breakthrough where "the light bulb goes on" surrenders to illumination and its baby "Eureka factor" the birth of innovation. This is where, previous ideas congregate to match as a coined new idea. Needless to mention that, "everyone has a creative spark, but many factors can inhibit its ignition" says one writer. This encourages creativity 
ignition, and champion its success. In this way, new ideas which are weak creations can be developed and cultivated, within the right business culture. It goes with saying that, "There is no smoke without fire" hence riding on this assumption I would safely retort that, "There is no creative 'SPARK' without knowledge and available opportunity."

\subsection{Systems Approach Perspective (SAP)}

A "System" is a collection of interrelated and interacting components that work together in an organized manner to fulfill a specific purpose or function (Dandy et al., 2008). Further, Wasson (2008) retorts that a system is an integrated set of operable elements, each with explicitly defined and bounded capabilities, working synergistically to enable a user to satisfy mission-oriented operations needed in a prescribed operating environment with a specified outcome and probability of success. In this regard, knowledge-based creativity can be enhanced through System Analysis Approach (SAAP) as an analytical tool in determining the best course of action to a given situation or problem. In this respect, awareness of creativity will help interrogate conceptual and theoretical frameworks on various knowledge fields, hence help create new ideas in problem solving environment. Figure 3, illustrates necessary steps in Systems Analysis Approach. In order to exploit previous research advancement in performance measurement system, as a basis for any successful business or institution (Kertu, Haldma, \& Moeller, 2011), established that decision making and operations can be improved. Therefore, systems analysis application to knowledge-based creativity, presents a need to innovation diffusion measure since it can influence creative "SPARK." Notwithstanding, (Liu et al., 2008) in their research submitted that, performance evaluation within specific industries still demands understanding and so is direct measure of creativity development process. In this regard, knowledge must be processed, through control process tools in decision making, such as systems approach method to create new ideas. Figure 5, illustrates necessary steps in use of system analysis approach in respect to decision making process.

\subsection{Validation}

In respect to this present study, using systems analysis approach (SAAP), creativityis viewed as an abstract system, based on necessary steps (process) required

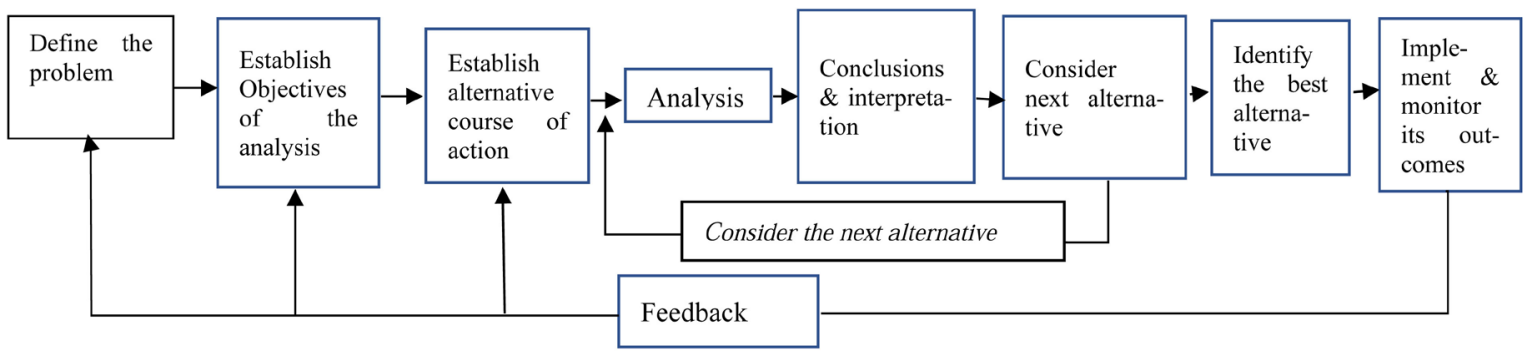

Figure 5. Basic steps in systems analysis (adopted from Samuel Labi, 2014). 
to develop creativity based on knowledge. Needless to mention that, creativity is an infusion process of different ideas to establish new ideas or perspectives. Therefore, to break the paradigms an exploratory dimension, risk taking and testing of new ideas is a very important step in the game of creativity and any failure is taken as part of the success process. Hence validation involves the verification and implementation in respect to the final selection of a new idea to solve a problem. In light of the aforementioned, adopted philosophy can stimulate creativity through value centered means of production and service delivery. Therefore, all knowledge-based creativity development process can be viewed as a system, in context of systems analysis approach based on domains of inquiry in systems theory focused on Figure 6 (Banathy, 1997) application

\subsection{Business Efficiency (Performance-Based Innovative Drive)}

For the purpose of this study, business efficiency is viewed in the context of performance-based innovative drive, in knowledge-based creativity. In light of the aforesaid, the immense wave of technological innovation has necessitated significant restructuring of business or organization (Grimm \& Smith, 2006). In the book entitled theory of economic development (Schumpeter, 1911), defined entrepreneurs, as change agents in society, and not just business creators. The success of a business depends on a performance measurement system to improve management decisions and operations (Kertu, Haldma, \& Moeller, 2011).

On the other hand, performance measures have been applied in various industries to improve operational efficiency, in Business, Finance, Health, education, Engineering, Military and Law enforcement but to mention a few. Further, appropriate measures in regard to controlling and improving organizational performance (Eltinary et al., 2013) cannot be under-estimated. Further, according to Eltinary et al. (2013) performance measurement is an important management control tool of any business in the current competitive environment. In this regard, performance measures in business help determine required innovative drive and ultimately knowledge-based creativity to ensure competitive edge

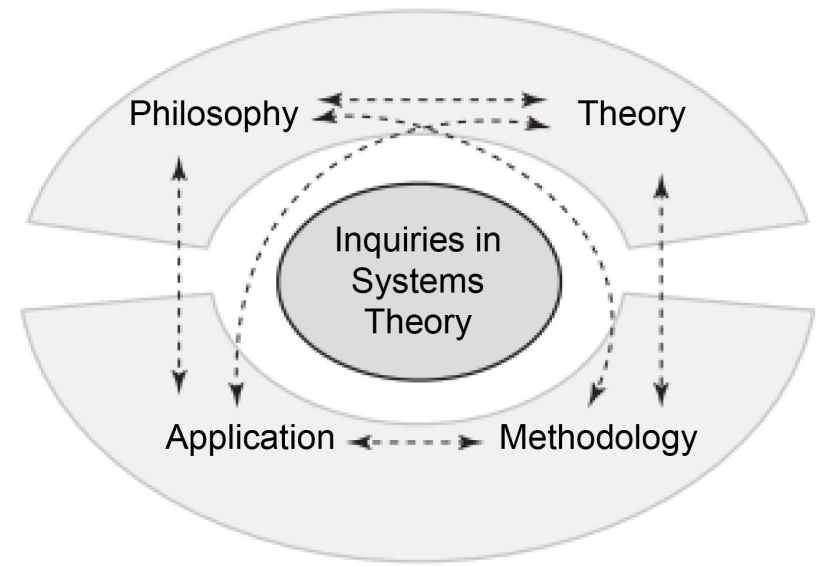

Figure 6. Domains of inquiry in system theory (adopted from Samuel Labi, 2014). 
in business. However, there can be no innovative drive without creativity based on knowledge.

\section{Results \& Analysis}

In respect to this present study, using systems analysis approach (SAAP); creativity is viewed as an abstract system, based on necessary steps (process) required to develop creativity based on knowledge. Needless to mention that, creativity is an infusion process of different ideas to establish new ideas or perspectives. Therefore, to break the paradigms, an exploratory dimension, risk taking and testing of new ideas is a very important step in the game of creativity and any failure is taken as part of the success. Table 3, illustrates a comparative creativity table based on Scarborough \& Cornwell (2016) and systems approach (Labi, 2014).

Group 1, was exposed to Systems Analysis Approach considering level of education and as a control group. Notwithstanding, Group 2, with high level of education was not exposed to the System Analysis Approach, but given same problem as the first group. Since both were given same problem or assignment, it was expected that they would come up with the same creative solution. However, the two groups presented different perspectives using different methods. The first group used systems approach, while it was observed that second group used more of brain storming.

In respect to this present study, using systems analysis approach (SAAP), it was observed that a systematic approach in creativity development process scored higher than the other method used. Further, groups were deliberately divided into two groups based on level of education. The assumption was that, first group of successful interpleural in Muchinga Province with no standard advanced education in business development be exposed to a systematic process using systems approach. It was observed that, group 1 score higher than group 2 on first level in respect to Philosophy at ten (10) and seven (7) respectively. However, the second group did well on measure of effectiveness than the first group still under philosophy. In terms of setting values and overall goals under

Table 3. Comparative table-creativity development process.

\begin{tabular}{lc} 
Seven step Process & Systems Analysis Approach \\
\hline $\begin{array}{l}\text { 1) Preparation } \\
\text { 2) Investigations }\end{array}$ & Establish objectives \\
3) Transformation & Analysis \\
4) Incubation & Conclusions \& interpretation \\
5) Illumination & Consideration of next alternative \\
6) Verification & Identify the best alternative \\
7) Implementation & Implementation \\
\hline
\end{tabular}


philosophy the two groups scored same. In regard to level 2 on theory using systems theory both groups scored equal points. However, the weighted distributed scoring perspective's value, overall goals, goals, objectives measure of effectives, differed on all fronts except on goals were both groups scored two (2). On level 3 , dealing with application it was observed that group 1 with high level of expectation scored higher than group two at thirteen (13) and ten (10) respectively. The first group had a better process control system and clear validation process. On level 4 dealing with methodology group 2 did better than group 1. It was noted that despite group 1 having a systematic procedure in creativity development process, knowledge gap in overall understanding of system theory concepts was still a challenge. Table 4, illustrates a 3-Point Matrix Scale based on Perspective with perspective's value, overall goals, goals, objectives measure of effectives the highest score is selected. And in this case perspective 1 which had scored 41 as opposed to perspective 2 which scored 36.

These results confirm our hypothesis, that choice of a new idea or creativity based on knowledge is influenced by decision process. Despite group 1, being less educated than group2, their exposure to System Analysis Approach showed a greater positive edge in creativity" SPARK" development. The first group first showed that they could easily develop different perspectives faster that highly educated group 2. Needless to mention that, creativity being an infusion process of different perspectives to establish new ideas demands a break into paradigms, through exploratory dimension, risk taking and testing of new ideas using Systems Analysis Approach (SAAP).

Suffice to mention that, the hierarchy of desired system starts with the basic

Table 4. Proposed non-numerical 3-point matrix scale (results).

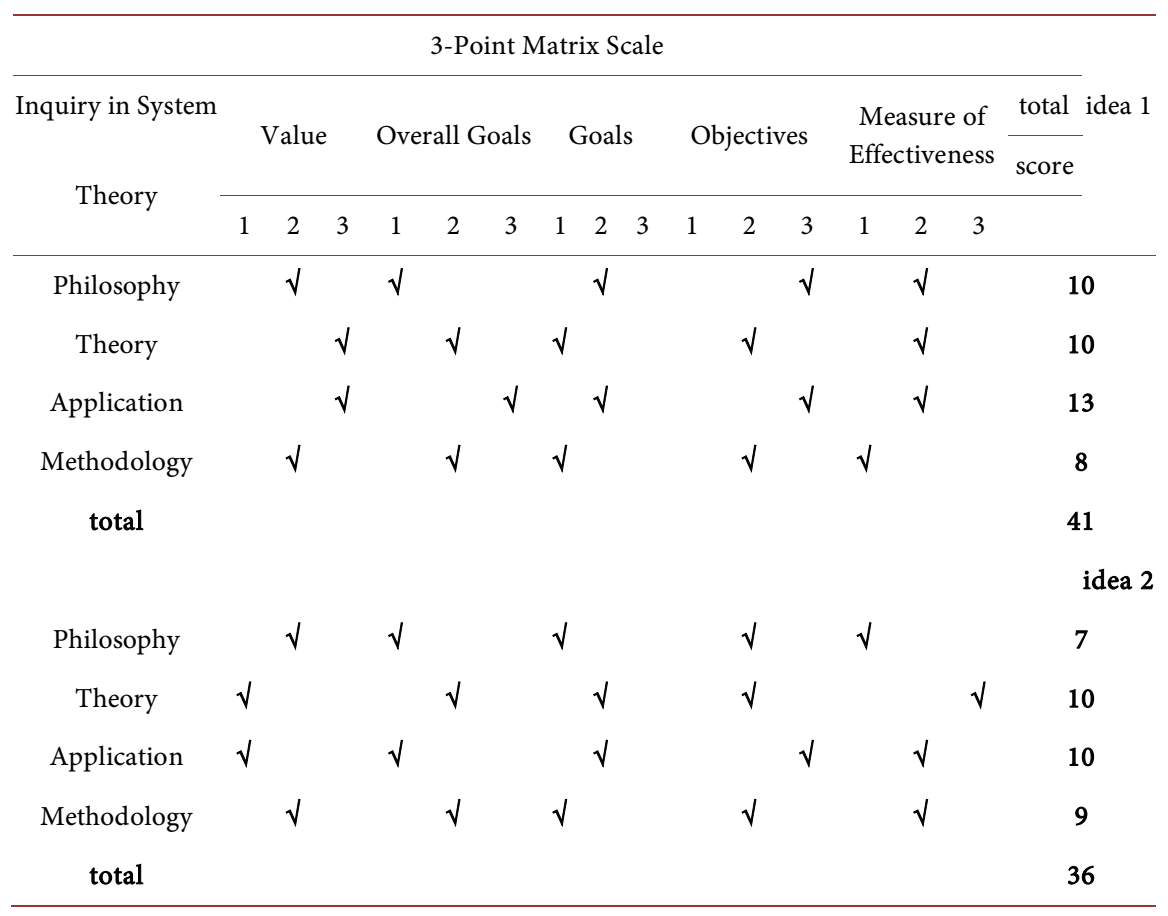


values of human society, followed by overall goals of effectiveness, efficiency and equity (Labi, 2014). Further, he retorted that the development of system goals and objectives is dependent on a hierarchy of desired system outcomes. In this respect, the desire to create new ideas must reflect expectations to societal needs or problems. Non-numerical weights are assigned based on level of satisfaction to desired system, in this case an abstract system (Idea). System analysis approach is pursued, to interrogate various view-points of CDP.

\subsection{Creativity Development Score-SAAP}

Based on scored results for perspective 1, which used System Analysis Approach (SAAP) it is observed that, its application to creativity development scored the highest compared to perspective's philosophy, Theory and methodology. This is consistence with wide application of SAAP in various engineering fields, commercial product development process, medical field in study of mental toughness. From the results, it's clear that, philosophy score has a great influence theory and application final adoption because of associated belief system to expected benefit in regard to creativity development method. Figure 7, illustrates the aforementioned as a structured approach system.

\subsection{Creativity Development Score-Brainstorming}

Based on results obtained using brainstorming by group2, it's clear that the perspective's method scored higher than perspective 1. However, due to low score on philosophy which has greater influence on theory and application, it is observed that perspective 2 scored less on application but had equal score on theory. Basically, theory which is field related knowledge shows that, there can be no creativity without knowledge. Therefore, the need to have a structured approach in creativity development process is seen through comparative based

\section{Creativity Development Score- SAAP}

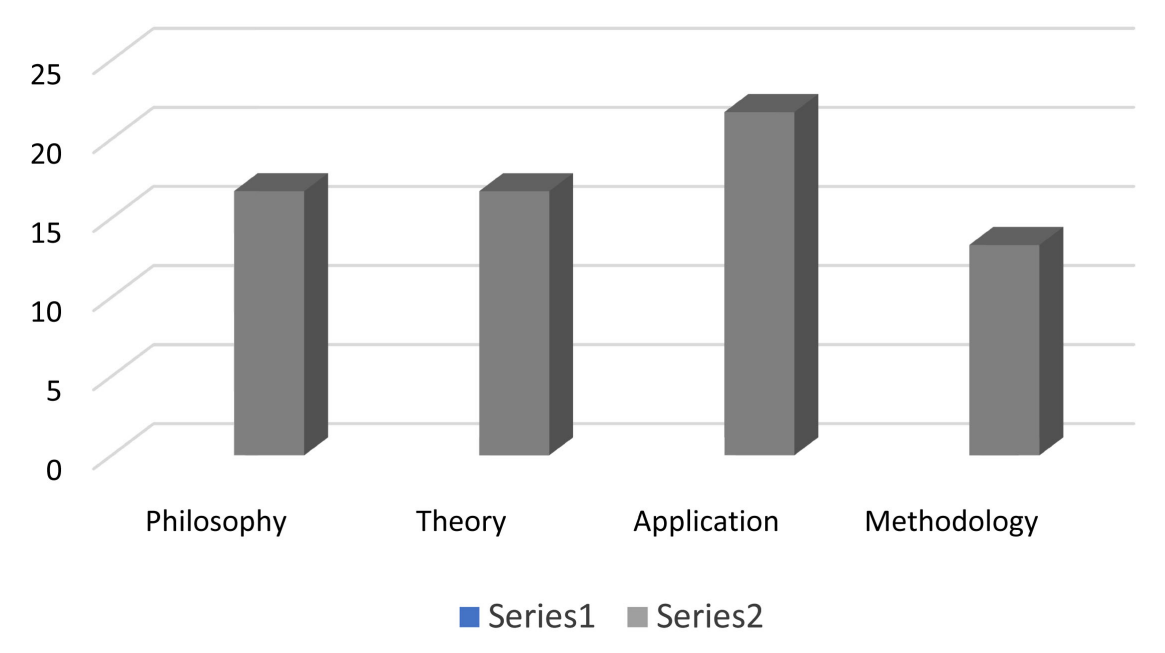

Figure 7. Creativity development score-SAAP. 
on perspective 1 and 2 results. Figure 8 illustrates the results using brainstorming.

\subsection{SAAP \& Brainstorming Comparison on Desired System Outcomes (Hierarchy)}

According to Labi (2014), the hierarchy of desired system outcomes viewed in our current research as a perspective (abstract system), and based on integration with four domains of system theory, each perspective's value, overall goals, goals, objectives and measure of effectiveness were compared. Based on perspective results in $1 \& 2$ in Figure $9 \&$ Figure 10 respectively showed that value-centeredness influenced overall choice of chosen perspective. It's also clear the in use of SAAP, decision process was mostly centered on value and this had greater effect on

\section{Creativity Development Score- Brainstorming}

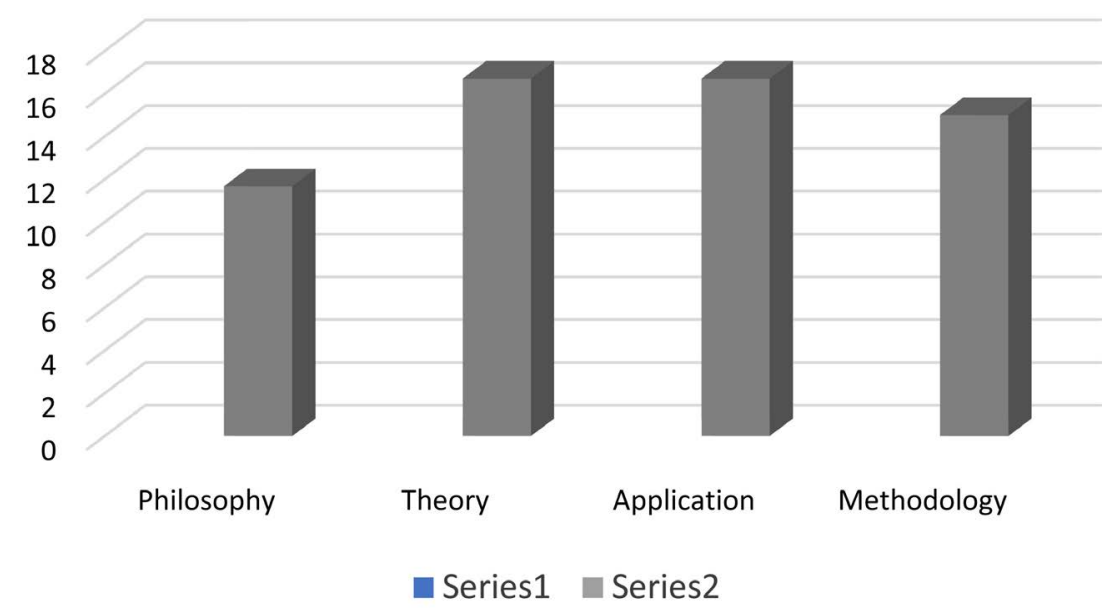

Figure 8. Creativity development score-brainstorming.

\section{Perspective Score 1}

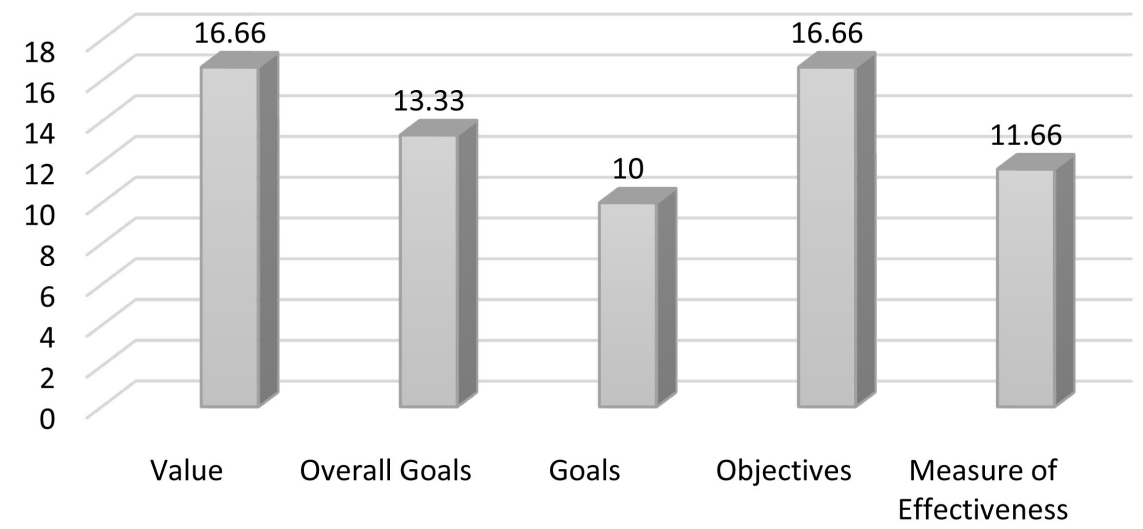

- Series1 Series2

Figure 9. Perspective score 1-SAAP. 


\section{Perspective Score 2}

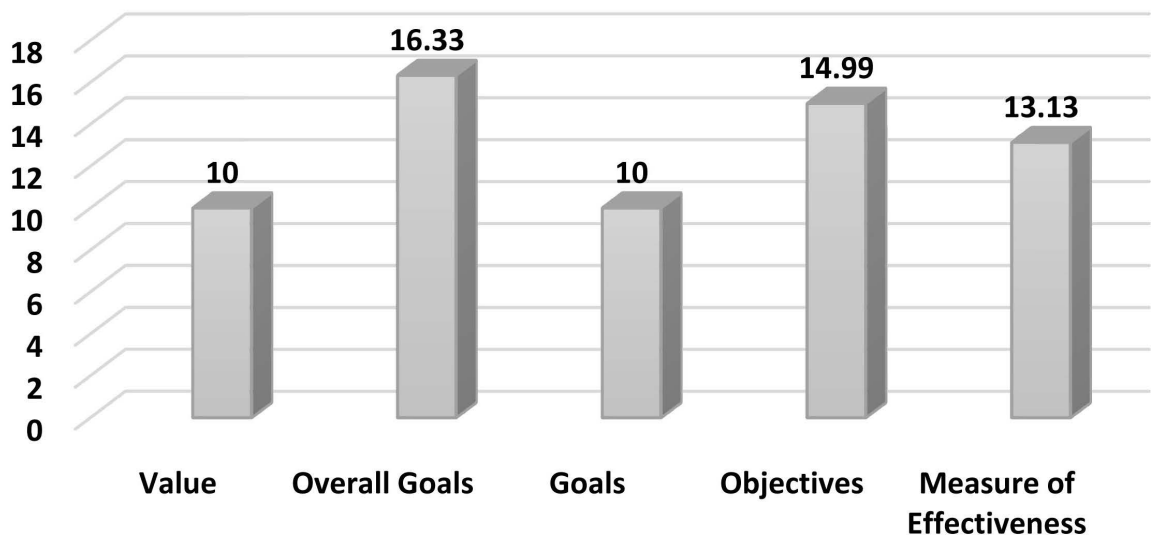

Series1 Series2

Figure 10. Perspective score 2-brainstorming.

coined overall goals, goals, objectives and measure of effectiveness. On the other hand, brainstorming, focused on method effectiveness but not value-centered witnessed lower over-all score to SAAP. Therefore, in creativity development process value analysis especially during problem identification and problem analysis plays critical role in quality of decision-making process. It is further noted that, in terms of overall goals setting both SAAP and Brainstorming did very well despite a slight score with former compared to the later. Notwithstanding, the results of goals setting showed equal score. However, in terms of setting objectives SAAP had higher score compared to Brainstorming. At this point it's important to realize that, objective alignment to creative "SPARK" is critical in validation process of the Creativity Development Process (CDP).

\subsection{Method \& Decision Performance (SAAP vis-a-vis Brainstorming)}

The present research adopted an integrated approach of systems inquiry (Banathy, 1997) and the hierarchy of desired system outcomes (Labi, 2014) viewed in our current research as a perspective (abstract system), in creativity development process. Suffice to say that; under aforementioned system theory philosophy and theory are associated with knowledge. In creativity development process, knowledge on need to underscore fundamental qualities of creativity such as originality, fluidity, flexibility and elaboration (Manriquez et al., 2005; Martin, 1980) is critical. Further, Sternberg and Lubart (1992) retorted that, "to be creative, you need to generate ideas that are relatively new, appropriate and of high quality." In this regard, the use of Systems Analysis Approach (SAAP) cannot be over-emphasized in realizing the aforementioned.

The development process of creativity based on knowledge followed systematic steps focused on systems approach for group 1, and brainstorming for group 
2. Since, the attention with this present research was to interrogate use of systems perspectives to enhance Creativity Development Process (CDP). The creativity development process for the control group followed the following steps: Problem definition, establishment of objectives, Analysis, Conclusion \& Interpretation, Consideration of next alternative and Implementation. In this regard, factors that influence creativity development process initiation are underscored and included: need, opportunity, differentiation, uniqueness, disruption and expected value analysis. Needless to say, that since creativity is the ability to create new ideas, the development process demands both implicit and explicit hierarchical system outcomes to effectively refine perspective selection.

Through application of decision process to analyze and select alternatives based on best values, overall goals, goals, objectives and measure of effectiveness, new ideas were generated. Notwithstanding, measure of effectiveness in this study does not refer to the applied method of decision process, but rather the selected perspective in regard to applicability. Consequently, fusion of diverse perspectives through a properly structured decision process presented better perspectives as indicated in Figure 11, where systems approach perspective scored higher than brainstorming method. However, brainstorming showed greater score on measure of effectiveness in regard to selected perspective as illustrated in comparison of the aforesaid measure in Figure 9 \& Figure 10. Further, Systems Approach showed overall best decision process as indicated in Figure 12, were group 1 scored higher than group 2 which used brainstorming. In terms of speed of generating new perspectives system approach perspective showed more generation through sub-group performance. However, despite brainstorming being slow in generating various perspectives within limited time, the measure of effectiveness of the few perspectives proved better than the systems approach style. Through, observation the level of education for group 2 compared to group 1, the analytical skills and knowledge based were wide. Therefore, we can conclude safely that if group 2 was exposed to systems approach, the

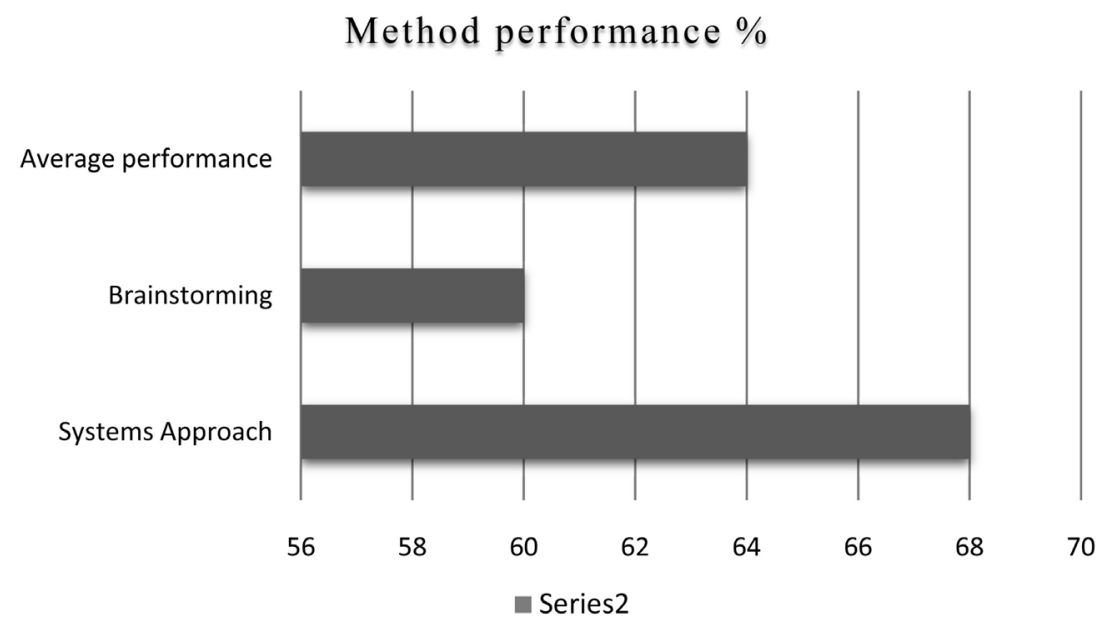

Figure 11. Method performance. 


\section{Decision Performance \%}

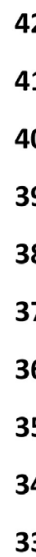

41
40
39
38
37
36
35
34
33
41

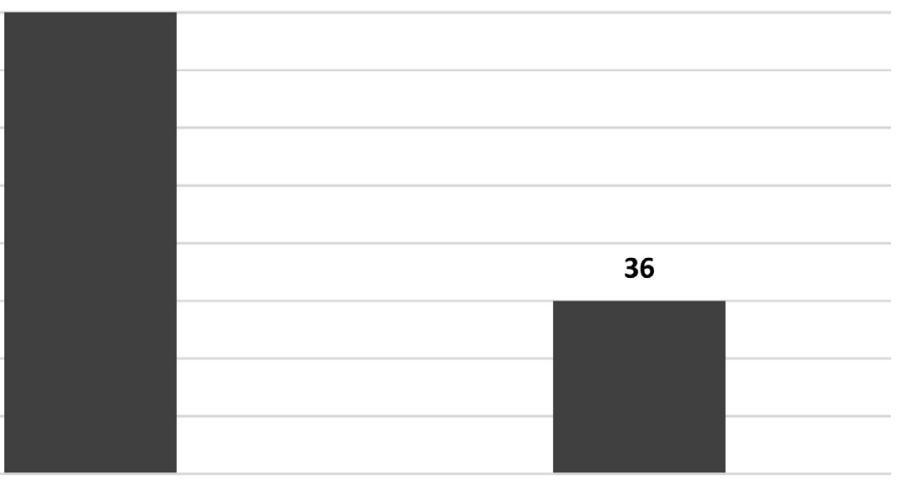

Group 1
Group 2

Figure 12. Decision performance.

likely wood of group 1 would have been slim. This where, retort that creativity development process can be enhanced through structured approach in decision process especially in application of systems analysis approach.

\subsection{Creativity Development Process (Seven Step Process \& SAAP)}

Needless to mention that, creativity being an infusion process of different perspectives to establish new ideas demands a break into paradigms, through exploratory dimension, risk taking and testing of new ideas using Systems Analysis Approach (SAAP). In view to development of creativity based on knowledge, using systems analysis approach, the decision process is consistence to Scarborough \& Cornwell (2016) and seven steps necessary to develop creativity which includes: preparation, investigation, transformation, incubation, illumination, verification and implementation. Table 4, illustrates the comparison on information collection but Systems analysis approach has an added advantage on decision making.

\section{Discussion}

The current research, in enhancement of Creativity Development Process (CDP) in business, through an eye of Systems Approach Perspective (SAP), will provide an analytical guide. Therefore, the need to develop a strong creative development process through systems approach analysis, demands a parallel drive in knowledge base. However, the increasing complexities in innovative diffusion and satisfaction measures still present a challenge on creativity based on knowledge in business performance. Therefore, discussing creativity development process without touching on innovative performance, would be an incomplete study tour on this subject. It's important to note that, knowledge on diffusion factors are likely to have both direct and indirect influence on innovation 
drive. On the other hand, one may ask if it's possible to have innovation without creativity. The vulnerability and speed in global social and economic development complexities, demands a well-structured creativity development process to strengthen innovative drive advancement.

\section{Conclusion}

According to Scarborough \& Cornwell (2016) he stated that "creativity the ability to develop new ideas and to discover new ways of looking at problems and opportunities." Therefore, a systems approach perspective in knowledge-based creativity, is proposed to enhance the determination of best course of action in regard to Creativity Development Process. This will provide a balanced analytical tool in interrogations and gaining rich insight into various viewpoints to a problem and subsequent selection of solutions.

Creativity, has played a major role in innovation advancement in various industries across the globe. Therefore, the importance of enhanced creativity development process (CDP), in creativity based on knowledge cannot be underestimated. Suffice to mention that, many challenges faced by society or industries have been solved through creativity and innovation drive. The current research, in enhancement of Creativity Development Process (CDP) in business, through an eye of Systems Approach Perspective (SAP), has shown consistency with seven steps (Scarborough \& Cornwell, 2016). Therefore, the need to develop a strong creative development process through systems approach analysis, demands a parallel drive in knowledge base. A "System" is a collection of interrelated and interacting components that work together in an organized manner to fulfill a specific purpose or function (Dandy et al., 2008). Further, Wasson (2008) retorts that a system is an integrated set of operable elements, each with explicitly defined and bounded capabilities, working synergistically to enable a user to satisfy mission-oriented operations needed in a prescribed operating environment with a specified outcome and probability of success.

However, the increasing complexities in innovative diffusion and satisfaction measures still present a challenge on creativity based on knowledge in business performance. Therefore, discussing creativity development process without touching on innovative performance, would be an incomplete study tour on this subject. It's important to note that, knowledge on diffusion factors is likely to have both direct and indirect influence on innovation drive. On the other hand, one may ask if it's possible to have innovation without creativity. The vulnerability and speed in global social and economic development complexities, demands a well-structured creativity development process to strengthen innovative drive advancement.

In order to have real creativity, advancement of knowledge wealth and human capital development are vital. In this regard, preparation of the mind for creative thinking based on knowledge is important and can be through formal education, on-the-job training, work experience, and other learning opportunities. The 
need to study the problem and understanding its basic components demands investigations. Needless to mention that, creative thinking has become a core business skill, and applying creative solutions to the problems and opportunities, provides innovative impetus necessary for any national survival and success. The immense wave of technological innovation has necessitated significant restructuring of business or organization (Grimm \& Smith, 2006). In advancing this current study, an Action Research is proposed to provide further understanding of knowledge-based creativity through systems approach perspective for promoting business efficiency.

In respect to this present study, using systems analysis approach (SAAP), it was observed that a systematic approach using systems analysis approach, in creativity development process scored higher than the other brainstorming method used by group 2. Dependent on hierarchy (Labi, 2014) of desired system outcomes view in our current research as a perspective (abstract system), based on four domains of system theory, an interrogation of the various perspectives of the main idea considering each perspective's value, overall goals, goals, objectives measure of effectives are perused. According to Eltinary et al. (2013) in quoting Olson \& Slate (2002) retorted that performance measurement is a significant component of management control process of any organization. In light of this, measurement of different viewpoints of the main idea, weighted using a non-numerical 3-point scale was used as an important control process in creativity development process.

Therefore, using the proposed 3-Point Matrix Scale based on Perspectives, with perspective's value, overall goals, goals, objectives measure of effectives and the highest score was selected. And in this case perspective 1 which had scored 41 as opposed to perspective 2 which scored 36 . Therefore, to break the paradigms into an exploratory dimension, risk taking and testing of new ideas is a very important step in the game of creativity and any failure is taken as part of the success process. Hence validation involved the verification and implementation in respect to the final selection of a new idea to solve a problem. In light of the aforementioned, adopted philosophy can stimulate creativity through value centered means of production and service delivery. The results based on 3-Point Matrix Scale, considering two perspectives developed by the two focus groups, confirmed our hypothesis that choice of a new idea or creativity based on knowledge is influenced by decision process.

\section{Recommendations}

In this current study, it is recommended that best methods to strengthen creativity development process in terms of analytical tools, be further researched. Focus, on role of measurement in creativity development analysis be extended to effect of value and satisfaction decision which affects innovation diffusion. In this regard, the extent of influence concerning innovation diffusion to creativity development process should be established. 


\section{Acknowledgements}

I wish to take this opportunity to say a million thanks to, Atlantic International University, USA, School of Business and Economics for allowing me to under-take this PhD Study in Project Management. Special thanks go to my Academic advisor for Dr Edward Lambert, AIU; My tutors Ms Linda Callazo and Ms Amaka Uzor, Ms Rina Lehnhoff under Admission, for their valuable contribution and guidance during my study tour. Great thanks go to the God of Major 1 for giving me wisdom in my studies.

\section{Conflicts of Interest}

The authors declare no conflicts of interest.

\section{References}

Banathy, B. H. (1997). A Taste of Systems. The Primer Project. http://www.rewciv.org/isss_Primer/asem04bb.html

Barbour, R. S. (2005). Making Sense of Focus Groups. Medical Education, 39, 742-750.

Collins (2009). Collins English Dictionary-Complete \& Unabridged(10th ed.). New York: Harper Collins.

Dandy, G., Walker, D., Daniell, T., \& Warner, R. (2008). Planning and Design of Engineering Systems (2nd ed.). London: Taylor \& Francis.

Eltinary, N. B., Masri, R., \& Chandran Govindaraju, V. G. R. (2013). Performance Measurement Usage in Developing Countries: The Case of Banking Sector-Sudan. Journal for Scientific \& Academic Publishing Management, 3, 488-490.

Fontana, A., \& Frey, J. H. (2005). The Interview: From Neutral Stance to Political Involvement. In N. K. Denzin, \& Y. S. Lincon (Eds.), The Sage Handbook of Qualitative Research (3rd ed., pp. 695-727). New York: Sage Publishing.

Frechtling, J., \& Sharp, L. (1997). User-Friendly Handbook for Mixed Method Evaluation. Alexandria, VA: National Science Foundation.

Grimm, C. M., Lee, H., \& Smith, K. G. (2006). Strategy as Action: Competitive Dynamics and Competitive Advantage. Oxford: Oxford University Press.

Kawalich, B. B. (2005). Participant Observation as Data Collection Method. Forum; Qualitative Research, 6, Article 43.

Lääts, K., Haldma, T., \& Moeller, K. (2011). Performance Measurement Patterns in Service Companies. Baltic Journal of Management, 6, 357-377. https://doi.org/10.1108/17465261111167993

Labi, S. (2014). Introduction to Civil Engineering Systems: A Systems Perspective to the Development of Civil Engineering Facilities. Hoboken, NJ: Wiley.

Liu, B., Fan, Y., \& Huang, S. (2008). A Service-Oriented Business Performance Evaluation Model and the Performance-Aware Service Selection Method. Concurrency \& Computation Practice \& Experience, 20, 1821-1836. https://doi.org/10.1002/cpe.1320

Manriquez, P., Carrasco, V. M., Navarro, N. M., Rivera, L., \& Pizarro, C. T. (2005). Creatividad y Profesores. Revista Iberoamericana de Education, 39, 1-5.

Martin, R. (1980). La Creatividad. Barcelona: Ediciones CEAC.

Meredith, J. R., Shafer, S. M., \& Mantel Jr., S. J. (2009). Project Management a Managerial Approach (7th ed.). Hoboken: John Wiley \& Sons, Inc. 
Oroszi, T. L. (2020). Traditional Organization Meeting Style Is Not Conducive to Group Decision-Making. Advances in Applied Sociology, 10, 115-127. https://doi.org/10.4236/aasoci.2020.104009

Rilling, J. K., \& Sanfey, A. G. (2011). The Neuroscience of Social Decision-Making. Annual Review of Psychology, 62, 23-48. https://doi.org/10.1146/annurev.psych.121208.131647

Sai, A. A. (2018). An Exploratory Study of Innovation Adoption in Estonia. Open Journal of Business and Management, 6, 857-889. https://doi.org/10.4236/ojbm.2018.64064

Scarborough, N. M., \& Cornwell, J. R. (2016). Essentials of Entrepreneurship and Small Business Management (8th ed.). Harlow, England: Pearson Education Limited.

Schumpeter, J. A. (1911). The Theory of Economic Development. An Inquiry into Profits, Capital, Credit, Interest, and the Business Cycle. Cambridge, MA: Harvard University Press.

Sekaran, U. (2003). Research Methods for Business. A Skill-Building Approach (4th ed.). Hoboken: John Wiley \& Sons, Inc.

Sternberg, R., \& Lubart, T. I. (1992). Creativity: Its Nature and Assessment. School Psychology International, 13, 243-253. https://doi.org/10.1177/0143034392133004

Wasson, C. (2008). Systems Analysis, Design, and Development. Hoboken, NJ: Wiley.

Wortman, M. S., \& Luthans (1969). Emerging Concepts in Management. New York: Macmillan.

Wu, H. Y., Wu, H. S., Chen, I. S., \& Chen, H. C. (2014). Exploring the Critical Influential Factors of Creativity for College Students. A Multiple Criteria Decision-Making Approach. Thinking Skills and Creativity, 11, 1-21.

http://www.sciencedirect.com/science/article/pii/s1871187113000606

https://doi.org/10.1016/j.tsc.2013.09.004 